\title{
Least-Squares Chebyshev Method for Solving Fractional Integro-diffrential Equations
}

\author{
Oyedepo Taiye ${ }^{1}$, Ayinde Muhammed Abdullahi ${ }^{2}$, Ishaq Adama Ajimati ${ }^{3}$, Adenipekun Adewale Emmanuel ${ }^{4}$ \\ ${ }^{1}$ Federal College of Dental Technology and Therapy, Enugu, Nigeria \\ ${ }^{2}$ Modibbo Adama University, Yola, Nigeria \\ ${ }^{3}$ AI-Hikmah University, Ilorin, Nigeria \\ ${ }^{4}$ Federal Polytechnic Ede, Osun, Nigeria
}

\begin{abstract}
The main purpose of this study gears towards finding numerical solution to fractional integro-differential equations. The technique involves the application of caputo properties and Chebyshev polynomials to reduce the problem to system of linear algebraic equations and then solved using MAPLE 18. To demonstrate the accuracy and applicability of the presented method some numerical examples are given. Numerical results show that the method is easy to implement and compares favorably with the exact results. The graphical solution of the method is displayed.
\end{abstract}

KEYWORDS: Fractional integro-differential equations; least squares; Chebyshev polynomials

\section{INTRODUCTION}

Fractional integro-differential equations has played a significant role in modelling of real world physical problems e.g the modeling of earthquake, reducing the spread of virus, control the memory behaviour of electric socket and many others. Fractional calculus is a field dealing with integral and derivatives of arbitrary orders, and their applications in science, engineering and other fields. The idea is from the ordinary calculus. According to [1-3], It was discovered by Leibniz in the year 1695 few years after he discovered ordinary calculus but later forgotten due to the complexity of the formula. Since most Fractional Integro-diffrential Equations (FIDEs) cannot be solved analytically, approximation and numerical techniques, therefore, they are used extensively.

Numerical solution to FIDEs in different fields has been a point of attraction for researchers in recent times. [4] employed Lagurre polynomials as basis functions for the solution of fractional Solving Fredholm integro-differential equations while [5] employed Bernstein polynomials as basis functions to approximate the solution of FIDEs. References [6 - 8] applied collocation techniques for solving FIDEs using different basis functions. [9] applied Sumudu transform method and Hermite Spectral collocation method for solving FIDEs. Author [10] introduced approximate solutions of Volterra-Fredholm integro-differential equations of fractional order. References [11 - 12] used Least - Squares method for the solution of FIDEs. [13-15] introduced numerical solution of fractional singular integro-differential equations by using Taylor series expansion and Galerkin method and a fast numerical algorithm based on the second kind of Chebyshev polynomials. The author in [16] applied numerical solution of Fredholm-Volterra fractional integrodifferential equation with nonlocal boundary conditions. Reference [17] employed Bernstein modified homotopy perturbation method for the Solution of Volterra fractional integro-differential equations. The objective of this work is to extend the application of the least squares and Chebyshev Polynomials to provide an approximate solution for fractional integro- differential equations taking the fractional derivative at equally spaced point of interval. The general form of the class of problem considered in this work is given as:

$$
\begin{aligned}
& D^{\alpha} u(x)=p(x) u(x)+f(x)+\int_{0}^{x} k(x, t) u(x) d t, \quad o \leq \\
& x, t \leq 1,
\end{aligned}
$$

With the following supplementary conditions: $u^{(j)}(0)=\delta_{j} j=0,1,2, \ldots, m-1, m-1<\alpha \leq m, m \in, N$ (2)

Where $D^{\alpha} u(x)$ indicates the $\propto$ th Caputo fractional derivative of $u(x) ; p(x), f(x)$,

$K(x, t)$ are given smooth functions, $\delta_{j}$ are real constant, $x$ and $t$ are real variables varying $[0,1]$ and $u(x)$ is the unknown function to be determined.

\section{SOME RELEVANT BASIC DEFINITIONS}

Definition 1: Riemann - Lowville fractional integral is defined as [18]:

$$
J^{\alpha} f(x)=\frac{1}{\Gamma(\alpha)} \int_{0}^{x} \frac{f(x)}{(x-t)^{1-\alpha}} d t, \alpha>0, x>0,
$$

denotes the fractional integral of order $\propto$ 
Definition 2: The Caputor Factional Derivative is defined as [18]:

$D^{\alpha} f(x)=\frac{1}{\Gamma(\mathrm{n}-\alpha)} \int_{0}^{x}(x-s)^{n-\alpha-1} f^{m}(s) d s$

Where $m$ is a positive integer with the property that $n-1<$ $\propto<n$

For example if $0<\propto<1$ the caputo fractional derivative is $D^{\alpha} f(x)=\frac{1}{\Gamma(1-\alpha)} \int_{0}^{x}(x-s)^{-\alpha} f^{1}(s) d s$

Hence, we have the following properties:

(1) $J^{\alpha} J^{v} f=j^{\alpha+v} f, \alpha, v>0, f \in C_{\mu}, \mu>0$

(2) $J^{\alpha} x^{\gamma}=\frac{\Gamma(\lambda+1)}{\Gamma(\alpha+\gamma+1)} x^{\alpha+\gamma}, \alpha>0, \gamma>-1, x>0$

(3) $J^{\alpha} \quad D^{\alpha} f(x)=f(x)-\sum_{k=0}^{n-1} f^{k}(0) \frac{x^{k}}{k !}, \quad x>$ $0, n-1<\alpha \leq n$

(4) $D^{\alpha} J^{\alpha} f(x)=f(x), \quad x>0, n-1<\alpha \leq n$,

(5) $D^{\alpha} C=0, C$ is the constant,

(6) $\left\{\begin{array}{lr}0, & \beta \in N_{0}, \beta<[\alpha], \\ D^{\alpha} x^{\beta}=\frac{\Gamma(\beta+1)}{\Gamma(\beta-\alpha+1)} x^{\beta-\alpha}, & \beta \in N_{0}, \beta \geq[\alpha],\end{array}\right.$

Where $[\alpha]$ denoted the smallest integer greater than or equal to $\alpha$ and $N_{0}=\{0,1.2, \ldots\}$

Definition 3: The Chebyshev polynomials [12] of degree $n$ over $[0,1]$ is defined by the relation

$Q_{m}(x)=\cos \left[\sin ^{-1}(2 x-1)\right]$

and the recurrence relation is given

$Q^{*}{ }_{m+1}(x)=2(2 x-1) Q_{m}^{*}(x)-Q_{m-1}^{*}(x)$,

$m \geq 1$

Where $\quad Q^{*}{ }_{0}(x)=1, \quad Q^{*}{ }_{1}(x)=2 x-1$

Definition 4: Chebyshev polynomials[12]: A linear combination Chebyshev basis polynomials:

$$
u_{m}(x)=\sum_{j=0}^{m} a_{j} Q_{j}^{*}(x)
$$

is the Bernstein polynomials of degree $\mathrm{n}$ where $a_{j,} j=$ $0,1,2, \ldots \ldots$. are constants.

\section{Demonstration of Least Squares Chebyshev Method (LSCM)}

The Least Squares Chebyshev Method is based on approximating the unknown function $u(x)$ in (1) by assuming an approximate solution of the form defined in (8). Consider equation (1) operating with $J^{\propto}$ on both sides as follows:

$$
\begin{aligned}
& J^{\propto} D^{\alpha} u(x)=J^{\propto} f(x)+J^{\propto}\left(\int_{0}^{x} k(x, t) u(t) d t\right) \\
& u(x)=\sum_{k=0}^{n-1} u^{k}(0) \frac{x^{k}}{k !}+J^{\propto} f(x)+ \\
& J^{\propto}\left[\int_{0}^{x} k(x, t) u(t) d t\right]
\end{aligned}
$$

Substituting (8) into (9) gives

$\sum_{j=0}^{m} a_{j} Q^{*}{ }_{j}(x)=\sum_{k=0}^{n-1} u^{k}(0) \frac{x^{k}}{k !}+J^{\propto} f(x)+$

$J^{\propto}\left[\int_{0}^{x} k(x, t) \sum_{j=0}^{m} a_{j} Q^{*}{ }_{j}(t) d t\right]$

Hence, the residual equation is obtained as

$$
\begin{gathered}
R\left(a_{0}, a_{1}, \ldots \ldots \ldots, a_{n}\right)= \\
\left\{\sum_{k=0}^{n-1} u^{k}(0) \frac{x^{k}}{k !}+J^{\propto} f(x)+\right. \\
\left.J^{\propto}\left[\int_{0}^{x} k(x, t) \sum_{j=0}^{m} a_{j} Q^{*}{ }_{j}(t) d t\right]\right\}
\end{gathered}
$$$$
\sum_{j=0}^{m} a_{j} Q^{*}{ }_{j}(x)-
$$

Let

$$
S\left(a_{0}, a_{1}, \ldots \ldots, a_{m}\right)=\int_{0}^{1}\left[R\left(a_{0}, a_{1}, \ldots \ldots, a_{m}\right)\right]^{2} w(x) d x
$$

Where $w(x)$ is the positive weight function defined in the interval, $[\mathrm{a}, \mathrm{b}]$. In this work,

we take $w(x)=1$ for simplicity. Thus,

$$
\begin{aligned}
& S\left(a_{0}, a_{1}, \ldots \ldots \ldots, a_{m}\right)=\int_{0}^{1}\left\{\sum_{j=0}^{m} a_{j} Q^{*}{ }_{j}(x)-\right. \\
& \left\{\sum_{k=0}^{m-1} u^{k}(0) \frac{x^{k}}{k !}+J^{\propto} f(x)+\right. \\
& \left.\left.\left[\int_{0}^{x} k(x, t) \sum_{j=0}^{m} a_{j} Q^{*}{ }_{j}(t) d t\right]\right\}\right\}^{2} d x
\end{aligned}
$$

In order to minimize equation (15), we obtained the values of $a_{j}(j \geq 0)$ by finding

the minimum value of $S$ as:

$$
\frac{\partial s}{\partial a_{j}}=0, j=0,1,2 \ldots, m
$$

Applying (15) on (14), we have

$$
\begin{aligned}
\int_{0}^{1}\left\{\sum_{j=0}^{m} a_{j} Q^{*}{ }_{j}(x)\right. & -\left\{\sum_{k=0}^{n-1} u^{k}(0) \frac{x^{k}}{k !}+J^{\propto} f(x)\right. \\
& \left.\left.+J^{\propto}\left[\int_{0}^{x} k(x, t) \sum_{i=0}^{m} a_{j} Q^{*}{ }_{j}(t) d t\right]\right\}\right\} d x \\
& \times \\
\int_{0}^{1}\left\{T^{*}{ }_{j}(x)\right. & \left.-J^{\propto}\left(\int_{0}^{x} k(x, t) T^{*}{ }_{j}(t) d t\right)\right\} d x
\end{aligned}
$$

Thus, (16) is then simplified for $j=0,1, \ldots n$ to obtain $(m+$ 1) algebraicsystem of equations in $(m+1)$ unknown $a^{\prime}{ }_{i} \mathrm{~s}$ which are then put in matrix form as follow:

A

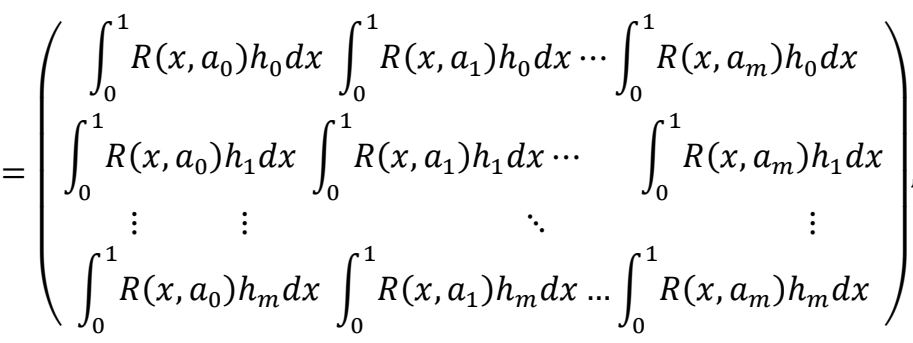

$$
\begin{aligned}
& B=\left(\begin{array}{c}
\int_{0}^{1}\left[J^{\propto} f(x)+\sum_{k=0}^{n-1} u^{k}(0) \frac{x^{k}}{k !}\right] h_{0} d x \\
\int_{0}^{1}\left[J^{\propto} f(x)+\sum_{k=0}^{n-1} u^{k}(0) \frac{x^{k}}{k !}\right] h_{1} d x \\
\vdots \\
\int_{0}^{1}\left[J^{\propto} f(x)+\sum_{k=0}^{n-1} u^{k}(0) \frac{x^{k}}{k !}\right] h_{m} d x
\end{array}\right)
\end{aligned}
$$

Where

$$
\begin{gathered}
h_{j}=Q^{*}{ }_{j}(x)-J^{\propto}\left[\int_{0}^{x} k(x, t) Q^{*}{ }_{j}(t) d t\right], \quad j=0,1, \ldots, m \\
R\left(x, a_{j}\right)=\sum_{i=0}^{m} a_{i} Q^{*}{ }_{j}(x)-J^{\propto}\left[\int_{0}^{x} k(x, t) \sum_{i=0}^{m} a_{i} Q^{*}{ }_{j}(t) d t\right], \\
j=0,1, \ldots, m
\end{gathered}
$$

The $(m+1)$ linear equations are then solved to obtain the unknown constants $a_{j}(j=0(1) m)$, which are then substituted back into the assumed approximate solution to give the required approximation solution. 


\section{NUMERICAL EXAMPLES}

In this section, the above technique is implemented on some problems. The problems are then solved via the Chebyshev polynomials as basis functions. The problems are then solved to illustrate the accuracy and efficiency of the proposed method using Maple 18.

Example 1: Consider the following fractional Integrodifferential [4]

$D^{\alpha} u(x)=f(x)+\int_{0}^{1} x t u(t) d t$

where

$f(x)=1-\frac{1}{3} x$

Subject to $u(0)=0$

Here, (18) is solved by applying $J^{\alpha}$ on both sides of (18) and substituting (8) into (18) to have

$J^{\alpha}\left[D^{\alpha} \sum_{j=0}^{m} a_{j} Q^{*}{ }_{j}(x)\right]=J^{\alpha}[f(x)]+$

$J^{\alpha}\left[\frac{1}{4} \int_{0}^{1} x t \sum_{j=0}^{m} a_{j} Q^{*}{ }_{j}(t) d t\right]$

Applying fractional integral operator on (20)

and simplifying further, where $m=2$ and $\propto=1$ gives

$a_{0}+(2 x-1)+a_{2}\left(8 x^{2}-8 x+1\right)-x+\frac{1}{6} x^{2}-$

$0.25 a_{0} x^{2}-0.08333333335 a_{1} x^{2}+$

$0.08333333335 a_{2} x^{2}=0$

Hence, the residual equation is defined as:

$R\left(a_{0}, a_{1}, a_{2}\right)=a_{0}+(2 x-1)+a_{2}\left(8 x^{2}-8 x+1\right)-$ $x+\frac{1}{6} x^{2}-0.25 a_{0} x^{2}-0.08333333335 a_{1} x^{2}+$

$0.08333333335 a_{2} x^{2}$

Let

$$
S\left(a_{0}, a_{1}, a_{2}\right)=\int_{0}^{1}\left[R\left(a_{0}, a_{1}, a_{2}\right)\right]^{2} w(x) d x
$$

Where $w(x)$ is has been defined above. Thus,

$S\left(a_{0}, a_{1}, a_{m}\right)=a_{0}+(2 x-1)+a_{2}\left(8 x^{2}-8 x+1\right)-x+$ $\frac{1}{6} x^{2}-0.25 a_{0} x^{2}-0.08333333335 a_{1} x^{2}+$ $0.08333333335 a_{2} x^{2}$

$\int_{0}^{1}\left[a_{0}+(2 x-1)+a_{2}\left(8 x^{2}-8 x+1\right)-x+\frac{1}{6} x^{2}-\right.$

$0.25 a_{0} x^{2}-0.08333333335 a_{1} x^{2}+$

$\left.0.08333333335 a_{2} x^{2}\right]^{2} d x$

In order to minimize (15), applying (16) on (25) and integrating with respect to $\mathrm{x}$ over the interval $[0,1]$ to give three system of equations with three unknown constants $a_{i}(i=0,1,2)$. Solving these equations, the following constants were obtained as: $a_{0}=0.5, a_{1}=0.5$ and $a_{2}=0$. Substituting the values back into (8) to get the approximate solution as: $u(x)=x$ which is the same as exact solution when $\propto=1$. Bernstein polynomials was used as basis functions to find the numerical solution of similar problem by [4]. The approximate solution was found at $\mathrm{N}=3$ as:

$u(x)=0.0024(1-x)^{3}+0.32 \times 3 x(1-x)+0.712 \times$ $3 x^{2}(1-x)+0.841 x^{2}$

Looking at the outcomes of the results, it tends to be said that our method performed more accurately since the exact solution is found.
Following the same procedure for $\propto=0.9, \propto=0.8, \propto=$ $0.7, \propto=0.6, \propto=0.5, \propto=0.4, \propto=0.3, \propto=0.2$ and $\propto=0.1$

$u(x)=0.01588902294+1.150959953 x-$

$0.1157895773 x^{2}$

$u(x)=0.04003025921+1.297646180 x-$ $0.2390914991 x^{2}$

$u(x)=0.0750324633+1.430521126 x-$

$0.3639375006 x^{2}$

$u(x)=0.1241382263+1.536726288 x-$

$0.4816550418 x^{2}$

$u(x)=0.1912818494+1.599296945 x-$

$0.5801507126 x^{2}$

$u(x)=0.2812494474+1.596242159 x-$

$0.6430517126 x^{2}$

$u(x)=0.3997678105+1.499487792 x-$

$0.648689190 x^{2}$

$u(x)=0.5536115751+1.273699667 x-$

$0.5689149551 x^{2}$

$u(x)=0.7506771300+0.8750423506 x-$

$0.3677620782 x^{2}$

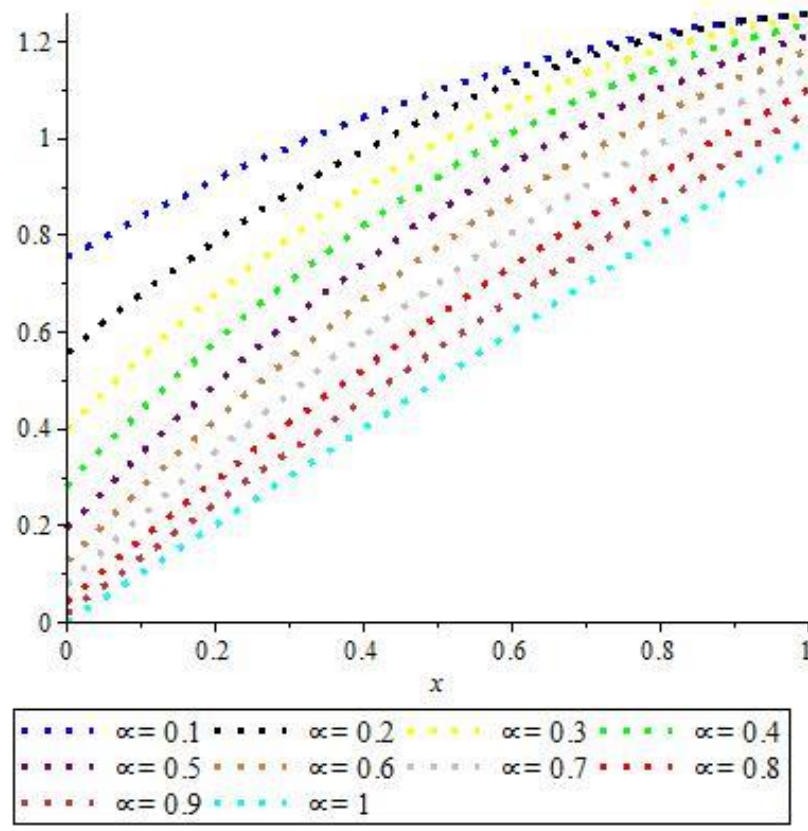

Figure 1: Showing the graphical behaviour of the approximation solutions of example 1

Example 2: Consider the following fractional Integrodifferential [19]

$D^{\propto} u(x)=f(x)+\int_{0}^{1} x t u(t) d t$,

where

$f(x)=1-e^{x}$

Subject to $u(0)=0$. Solving (27), following the same procedure above, we take $\propto=1, \propto=0.9, \propto=0.8, \propto=0.7, \propto$ $=0.6, \propto=0.5, \propto=0.4, \propto=0.3, \propto=0.2$ and $\propto=0.1$. The following approximate solutions are obtained. 

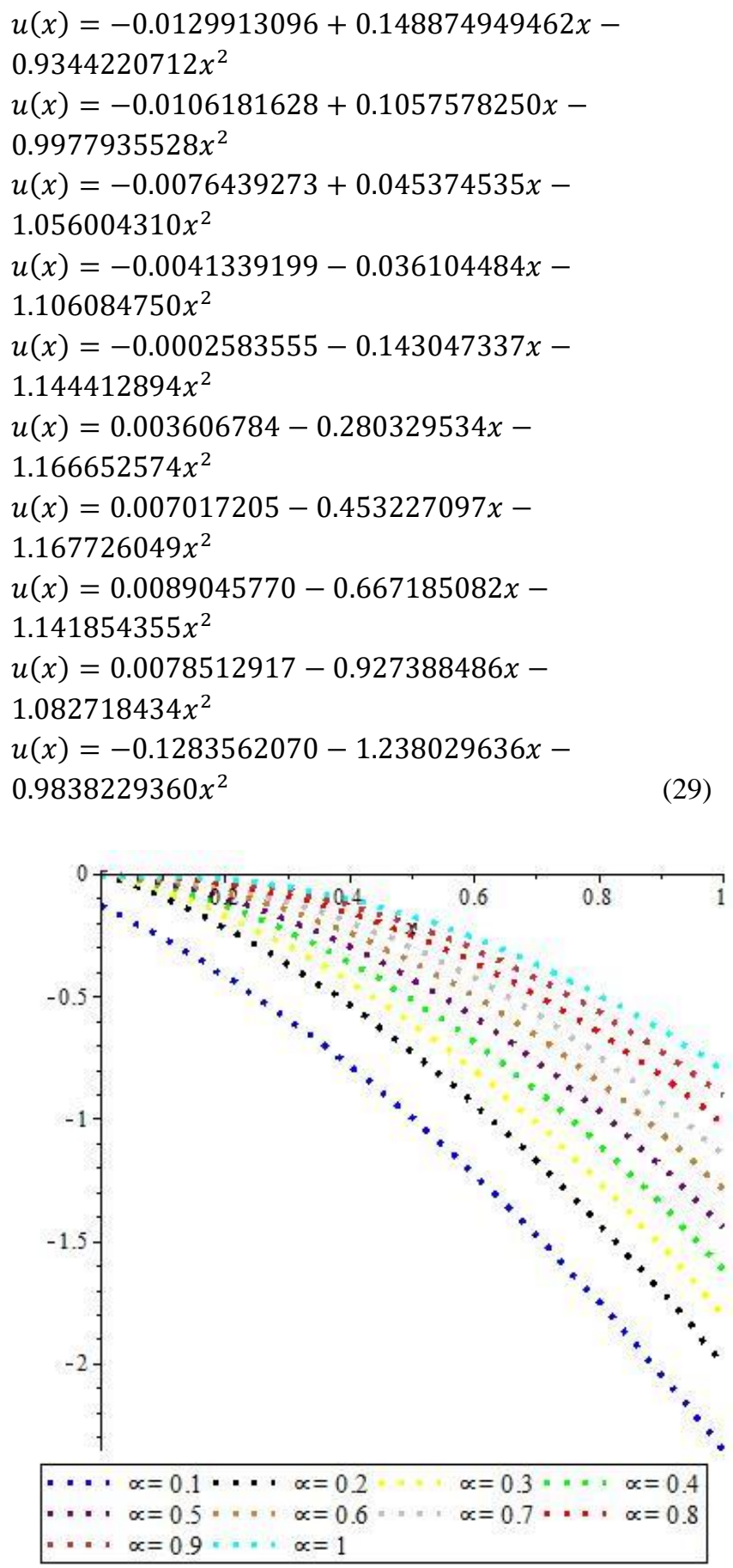

Figure 2: Showing the graphical behavior of the approximation solutions of example 2

\section{CONCLUSION}

In this study, the Chebyshev polynomials together with Caputo properties are used to find the solution of FIDEs. There is a high rate of convergence of the approximate solutions to the exact solutions. Specifically, the performance of the proposed method was compared with existing results in the literature and are found to be more efficient in the terms of accuracy. Also, it is pleasing to note that the Chebyshev polynomials and the method used displayed a good behaviour on the graph when set into a control system at equal spaced point of fractional derivatives. It is also observed that the number of iterations needed in solving the problems using the proposed method is few and with lower values of $\mathrm{M}$ (the degree of the approximant).

Acknowledgement: Authors thank those who contributed to write this article and give some valuable comments.

Conflicts of Interest: The authors declare that they have no conflicts of interest to report regarding the present study.

\section{REFERENCES}

1. Adam, L., "Fractional Calculus: history, definition and application for the engineer," Department of Aerospace and Mechanical Engineering University of Notre Dame, IN 46556, U.S.A. (2004).

2. Awawdeh, F., Rawashdeh, E. A. and Jaradat, H.M., "Analytic solution of fractional integro-differential equations," Annals of the University of CraiovaMathematics and Computer Science Series (2011), vol. 38, no. 1, pp. 1-10.

3. Caputo, M., "Linear models of dissipation whose $\mathrm{Q}$ is almost frequency Independent," Geophysical Journal International (1967), vol. 13, no. 5, pp.529 539.

4. Dr.Osama H. Mohammed and Sarmad A. Altaie "Approximate solution of Fractional IntegroDifferential Equations by using Bernstein polynomials, Engineering and Technology Journal (2012), vol. 30 no. 8, pp. 1362-1373.

5. Aysegul, D. and Dilek V. B., "Solving fractional Fredholm integro-differential equations by Laguerre polynomials", Sains Malaysiana (2019), Vol. 48, no. 1 ,pp. 251-257.

6. Dilkel,V. B. and Aysegül, D.,"Applied collocation method using Laguerre polynomials as the basis functions," Advances in difference equations a springer open journal (2018), pp. 1- 11.

7. Ma, X. and Huang, C., "Spectral collocation method for linear fractional integro- differential equations," Applied Mathematical Modelling (2014), vol. 38, no. 4, pp. 1434-1448

8. Ma, X, and Huang, C "Numerical solution of fractional integro-differential equations by a hybrid collocation method," Applied Mathematics and Computation (2013), vol. 219, no. 12, pp. 6750 6760.

9. Amr, Y. A., Mahdy, M. S. and Youssef, E. S. M., "Solving fractional integro-differential equations by using Sumudu transform method and Hermite spectral collocation method," Computers, Materials and Continua (2018), vol. 54, no. 2, pp. 161-180.

10. Alkan, S. and Hatipoglu, V. F., "Approximate solutions of Volterra-Fredholmintegro-differential equations of fractional order," Tbilisi Mathematical Journal (2017), vol. 10, no. 2, pp.1-13.

11. Mahdy A. M. S. and Mohamed, R. M. H., "Numerical studies for solving system of linear fractional integro-differential equations by using least squares method and shifted Chebyshev polynomials," Journal of Abstract and Computational Mathematics (2016), vol. 1, no. 24, pp. 24-32, 2016.

12. Mohammed, D. Sh., "Numerical solution of 
fractional integro-differential equations by least squares method and shifted Chebyshev polynomial," Mathematical Problems in Engineering (2014), Article ID 431965, no. 1, pp. 1-5.

13. Mohammed, D. Sh., "Numerical solution of fractional singular integro-differential equations by using Taylor series expansion and Galerkin method," Journal of Pure and Applied Mathematics: Advances and Applications (2014), vol. 12 no. 2, pp. 129-143.

14. Nemati, S., Sedaghatb, S. and Mohammadi, I., "A fast numerical algorithm based on the second kind Chebyshev polynomials for fractional integrodifferential equations with weakly singular kernels,",Journal of Computational and Applied Mathematics (2016), vol. 308, pp. 231-242.

15. Yi, M. and Huang, J., "CAS wavelet method for solving the fractional integro-differential equation with a weakly singular kernel," International Journal of Computer Mathematics (2015), vol. 92, no. 8, pp. 1715-1728.

16. Setia, A., Liu, Y. and A. S. Vatsala, A. S. "Numerical solution of Fredholm-Volterra Fractional integrodifferential equation with nonlocal boundary conditions," Journal of Fractional Calculus and Applications (2014), vol. 5, no. 2, pp. 155-165.

17. Oyedepo, T., Taiwo, O. A., Adebisi, A. F., Raji, M. T., Ajisope M. O. et al, "Bernstein modified Homotopy perturbation method for the solution of Volterra Fractional integro-differential equations," Pasifi Journal of Science and Technology (2021), vol. 22, no. 1, pp., $30-36$.

18. C. Edwards, "Math 312 Fractional calculus final presentation,"[video] Available at: https://www.youtube.com/watch?v=CsJa3XiOmfs [ Accessed 20 Sep. 2018].

19. Nanware, J. A., Goud, P. M. and Holambe, T. L., "Solution of fractional integro-differential equations by Bernstein polynomials," Malaya Journal of Matematik (2021), vol. S, no. 1, pp. 581-586. 